\title{
A Defect in Sodium-dependent Amino Acid Uptake in Diabetic Rabbit Peripheral Nerve

\author{
Correction by an Aldose Reductase Inhibitor or myo-Inositol Administration
}

\author{
Douglas A. Greene, Sarah A. Lattimer, Patricia B. Carroll, John D. Fernstrom, and David N. Finegold \\ Diabetes Research Laboratories of the Department of Medicine, and the Western Psychiatric Institute and Clinic, School of Medicine, \\ University of Pittsburgh, Pittsburgh, Pennsylvania 15261; and the Diabetes Research and Training Center and the \\ Department of Internal Medicine of the University of Michigan, Ann Arbor, Michigan 48109
}

\begin{abstract}
A myo-inositol-related defect in nerve sodium-potassium ATPase activity in experimental diabetes has been suggested as a possible pathogenetic factor in diabetic neuropathy. Because the sodium-potassium ATPase is essential for other sodiumcotransport systems, and because myo-inositol-derived phosphoinositide metabolites regulate multiple membrane transport processes, sodium gradient-dependent amino acid uptake was examined in vitro in endoneurial preparations derived from nondiabetic and 14-d alloxan diabetic rabbits. Untreated alloxan diabetes reduced endoneurial sodium-gradient dependent uptake of the nonmetabolized amino acid 2-aminoisobutyric acid by $>50 \%$. Administration of an aldose reductase inhibitor prevented reductions in both nerve myo-inositol content and endoneurial sodium-dependent 2-aminoisobutyric acid uptake. Myo-inositol supplementation that produced a transient pharmacological elevation in plasma myo-inositol concentration, but did not raise nerve myo-inositol content, reproduced the effect of the aldose reductase inhibitor on endoneurial sodiumdependent 2-aminoisobutyric acid uptake. Phorbol myristate acetate, which acutely normalizes sodium-potassium ATPase activity in diabetic nerve, did not acutely correct 2-aminoisobutyric uptake when added in vitro. These data suggest that depletion of a small myo-inositol pool may be implicated in the pathogenesis of defects in amino acid uptake in diabetic nerve and that rapid correction of sodium-potassium ATPase activity with protein kinase $C$ agonists in vitro does not acutely normalize sodium-dependent 2-aminoisobutyric acid uptake. (J. Clin. Invest. 1990. 85:1657-1665.) amino acid transport $\bullet$ amino acid metabolism • diabetic neuropathy • polyol pathway • sorbitol pathway
\end{abstract}

\section{Introduction}

Derangements in peripheral nerve metabolism resulting from insulin deficiency and/or hyperglycemia are associated with rapidly reversible abnormalities in nerve function in diabetic animals, and are thought to contribute to progressively less

Address reprint requests to Dr. Greene, Room 5570, MSRB-2, Box 0678, 1150 West Medical Center Drive, University of Michigan, Ann Arbor, MI 48109.

Received for publication 21 April 1989 and in revised form 5 September 1989.

J. Clin. Invest.

(c) The American Society for Clinical Investigation, Inc.

0021-9738/90/05/1657/09 \$2.00

Volume 85, May 1990, 1657-1665 reversible structurally based functional defects in animal and possibly human diabetic neuropathy (1-3). A reduction in neural free myo-inositol (MI) ${ }^{1}$ content that accompanies both acute spontaneous and experimentally induced diabetes in rodents appears to underlie a cascade of metabolic and functional defects in nerve, including reduced sodium-potassium ATPase activity and impulse conduction velocity $(2,3)$. In the acutely diabetic rat, these abnormalities are completely prevented or reversed by MI supplementation or aldose reductase inhibitors, both of which correct the fall in nerve MI level. Thus, increased polyol (sorbitol) pathway activity appears to contribute to MI depletion and its consequences in diabetic nerve (2). The MI-related decrease in sodium-potassium ATPase activity has been proposed as the basis for the early and reversible slowing of nerve conduction velocity and paranodal axonal swelling that are also reversed by aldose reductase inhibitors or MI supplementation in spontaneously diabetic BB rats (3). In vitro exposure to protein kinase $C$ activating phorbol esters or functionally related phosphoinositide-derived diacylglycerols acutely normalizes the MI-related defect in ouabain-sensitive ATPase activity in plasma membranes from diabetic rat sciatic nerve (4), as well as the diminished ouabain-sensitive respiration (5) and ATPase activity (6) in nerve preparations from alloxan diabetic rabbits, thereby suggesting that protein kinase $\mathrm{C}$ is a possible link between MI depletion and its associated sodium-potassium ATPase defect (2).

The relevance of these metabolic defects to the functional and structural abnormalities underlying human diabetic peripheral neuropathy (and perhaps other chronic complications of diabetes $[7,8]$ ) remains controversial. Aldose reductase inhibitors improve nerve conduction in nonneuropathic diabetic patients (9) and ameliorate the sorbitol accumulation $(10,11)$ and structural fiber abnormalities (10) in the peripheral nerves of neuropathic diabetic subjects. MI administration also appears to marginally improve nerve conduction in highly selected diabetic neuropathic patients (12-15), but not in more heterogeneous populations of diabetic subjects $(16,17)$. (The clinical efficacy of aldose reductase inhibitors or MI supplementation await large scale, long-term, randomized, controlled clinical trials with these drugs $[10,16,17])$. The conflicting and fragmentary reports of measurements of whole nerve MI content in diabetic humans $(11,18-21)$ and the reported lack of an effect thereon of aldose reductase inhibitor therapy $(11,21)$ has recently been used to argue that altered

1. Abbreviations used in this paper: AIB, 2-aminoisobutyric acid; BCAA, branched-chain amino acid; KRB, Krebs Ringer bicarbonate buffer; $K_{t}$, transport constant; MeAIB, 2-methyl-aminoisobutyric acid; MI, myo-inositol; PMA, phorbol myristate acetate; s, substrate concentration; $v$, uptake velocity; $V_{\max }$, maximum transport velocity. 
MI metabolism is irrelevant to the pathogenesis of diabetic neuropathy $(11,22,23)$. This view has been disputed $(24,25)$ partly on the basis of the fact that MI metabolism is now thought to be highly compartmentalized, and that depletion of a putative small metabolically labile MI pool by glucose during in vitro incubation of aortic intima-media preparations impairs tissue function (including sodium-potassium ATPase activity) in the absence of detectable changes in tissue MI content (26). One may therefore speculate that pathogenetically significant depletion or repletion in nerve MI could occur in diabetes without detectable alterations in tissue MI content. This communication reports that MI treatment corrects a sorbitol-related defect in amino acid transport in peripheral nerve without correcting the diminished nerve MI content, suggesting that nerve MI content may be an insensitive indicator of polyol pathway-induced defects in MI metabolism.

\section{Methods}

Experimental animal model. Male, white New Zealand rabbits weighing 1.5-2.0 kg were fed Wayne Rabbit Ration (Continental Grain Co., Chicago, IL) and fasted overnight before the study or the induction of alloxan diabetes as described previously (27). Alloxan monohydrate, $90 \mathrm{mg} / \mathrm{kg}$ i.v. (Sigma Chemical Co, St. Louis, MO) in $0.73 \%$ sodium chloride solution was administered by rapid injection to induce experimental diabetes. Nonfasting plasma glucose concentrations were measured $48 \mathrm{~h}$ later, and rabbits with values $<300 \mathrm{mg} / \mathrm{dl}$ were excluded. 2 wk thereafter, surviving animals were fasted overnight, and only rabbits with plasma glucose concentrations $\geq 300 \mathrm{mg} / \mathrm{dl}$ were studied. Nondiabetic saline-injected control animals were maintained for $14 \mathrm{~d}$ under similar environmental conditions (27). Sorbinil (Pfizer Central Research, Groton, CT) was suspended in water and administered by gavage daily at a dose of $15 \mathrm{mg} / \mathrm{kg}$ beginning $48 \mathrm{~h}$ after alloxan administration. MI was similarly administered daily by gavage at a dose of $100 \mathrm{mg} / \mathrm{kg}$.

In vitro peripheral nerve preparations. Endoneurial preparations were derived from the tibial division of the sciatic nerve as previously described in detail (28). After an overnight fast, diabetic and nondiabetic rabbits were sedated with diazepam, $2 \mathrm{mg} / \mathrm{kg}$ i.m. After induction of anesthesia (sodium pentabarbital $24-36 \mathrm{mg} / \mathrm{kg}$ i.v.), a defined segment of each sciatic nerve was surgically exposed, ligated, removed with preservation of hemostasis, and transferred to a $125-\mathrm{ml}$ erlenmeyer flask containing $70 \mathrm{mg}$ of collagenase (Type I; Sigma Chemical Co.) dissolved in $10 \mathrm{ml}$ of $4.5 \%$ dialyzed defatted bovine serum albumin (BSA) (Sigma fraction V powder) and $20 \mathrm{mM}$ glucose in KrebsRinger bicarbonate buffer medium (KRB), pH 7.4 equilibrated with $5 \% \mathrm{CO}_{2} / 95 \% \mathrm{O}_{2}$ at $37^{\circ} \mathrm{C}$. A separate portion of sciatic nerve proximal to the resected segment was removed and rapidly frozen in liquid nitrogen partially evacuated to its freezing point for analysis of MI content. After controlled digestion for 12-14 min, the ligated nerve segments were rinsed in $30 \mathrm{ml}$ of similar medium containing aprotinin, 200 kallikrein inactivation $\mathrm{U} / \mathrm{ml}$ (Trasylol; FBA Pharmaceuticals, New York). A branch-free length of the major fascicle of the tibial division of each ligated sciatic nerve segment was further ligated and microdissected to produce the endoneurial preparation (28).

Standard incubation medium for the endoneurial preparation was 4.5\% dialyzed defatted BSA and $5 \mathrm{mM}$ glucose in $\mathrm{KRB}, \mathrm{pH} 7.4$, equilibrated with $5 \% \mathrm{CO}_{2} / 95 \% \mathrm{O}_{2}$ as previously described (28). The endoneurial preparation was routinely equilibrated for $10 \mathrm{~min}$ in $3 \mathrm{ml}$ of standard medium in a Dubnoff metabolic shaker set at $88 \mathrm{cycles} / \mathrm{min}$ and $37^{\circ} \mathrm{C}$. The equilibrated endoneurial preparation was transferred to a new flask containing standard medium for incubation studies lasting as long as $2 \mathrm{~h}$. Phorbol myristate acetate (PMA) was prepared in dimethylsulfoxide (DMSO) $(300 \mu \mathrm{g} / \mathrm{ml})$ and $10 \mu \mathrm{l}$ added to the incubation flasks for a final PMA concentration of $2 \mathrm{nmol} / \mathrm{ml}$ for $1 \mathrm{~h}$ (controls received $10 \mu \mathrm{l}$ of DMSO alone). Other modifications in the incubation conditions for specific studies are described in the relevant portions of the text and figures.

2-Aminoisobutyric acid (AIB) uptake measurements. The preincubated endoneurial preparation was transferred to a new flask containing standard medium with $0.5 \mu \mathrm{Ci} / \mathrm{ml}$ of $1-\left[{ }^{14} \mathrm{C}\right]$ mannitol $45 \mathrm{mCi} /$ $\mathrm{mmol}$, and $\left.1 \mu \mathrm{Ci} / \mathrm{ml} \mathrm{[}{ }^{3} \mathrm{H}\right] \mathrm{AIB} 20 \mathrm{Ci} / \mathrm{mmol}$ (New England Nuclear, Boston, MA) to which unlabeled AIB or MeAIB was added to produce the specified concentrations. To assess the effects of sodium ion in the extracellular fluid on AIB uptake, sodium was replaced by equimolar concentrations of choline during the last $15 \mathrm{~min}$ of preincubation and during the exposure to isotopically labeled compounds. Sodium-dependent AIB uptake was defined as the difference in the intracellular uptake in the presence or absence of sodium under each experimental condition.

After brief, timed exposure to isotopically labeled medium the endoneurial preparation was rapidly rinsed in AIB-free standard medium, and frozen in liquid nitrogen. The frozen endoneurial preparation was rapidly weighed on a microbalance, powdered in liquid nitrogen, and homogenized in $6 \%$ perchloric acid. Incubation medium was quantitatively transferred to a volumetric flask and diluted to a 5-ml volume with water. Aliquots of diluted medium were deproteinized with equal volumes of $12 \%$ perchloric acid. After centrifugation at $2,500 \mathrm{~g}$ for $15 \mathrm{~min}$ at $4^{\circ} \mathrm{C}$, the supernatant of the tissue or medium perchloric acid extract was neutralized with $\mathrm{KOH}$ and recentrifuged. A 0.4-ml aliquot of the resulting supernatant was added to $15 \mathrm{ml}$ of Aquasol II (New England Nuclear) and counted for ${ }^{3} \mathrm{H}$ and ${ }^{14} \mathrm{C}$ in a liquid scintillation spectrometer (Packard Tri-Carb; Packard Instrument Co., Downers Grove, IL) with automatic external standard ratios to correct for quenching. Cross-over of ${ }^{14} \mathrm{C}$ and ${ }^{3} \mathrm{H}$ radioactivity was determined at varying automatic external standard ratios in vials containing varying amounts of acetone in water as a quenching agent. Recovery of $\left[{ }^{3} \mathrm{H}\right]$ AIB added to endoneurial homogenates was > 95\% on a volume basis when compared with recovery after neutralization and centrifugation of $\left[{ }^{3} \mathrm{H}\right] \mathrm{AIB}$ added to $6 \%$ perchloric acid. The extracellular tissue space in milliliter per gram wet weight was calculated as: (dpm ${ }^{14} \mathrm{C} / \mathrm{g}$ wet weight of tissue) $\div\left(\mathrm{dpm}{ }^{14} \mathrm{C} / \mathrm{ml}\right.$ of incubation medium). Tissue and medium ${ }^{3} \mathrm{H}$ was partially characterized by lyophilization and thin-layer chromatography. Recovery of ${ }^{3} \mathrm{H}$ from tissue or medium filtrates postincubation was $>95 \%$ after lyophilization compared to fresh $\left[{ }^{3} \mathrm{H}\right] \mathrm{AIB}$ suspended in $6 \%$ perchloric acid and neutralized with $\mathrm{KOH}$, suggesting that ${ }^{3} \mathrm{H}$ from AIB was not volatilized during tissue incubation. Tritiated AIB-containing incubation medium exposed to endoneurial preparations for $2 \mathrm{~h}$ also had no detectable ${ }^{3} \mathrm{H}_{2} \mathrm{O}$ by microdiffusion compared to a medium blank incubated without tissue. Water soluble ${ }^{3} \mathrm{H}$ from AIB-exposed endoneurial filtrates comigrated with $\left[{ }^{3} \mathrm{H}\right] \mathrm{AIB}$ when chromatographed on cellulose thin-layer plates developed in butanol/acetic acid/water (25:4:10). Endoneurial ouabain-sensitive uptake was essentially irreversible: there was no significant difference in the recovery of ${ }^{3} \mathrm{H}$ effluxed over $10 \mathrm{~min}$ into AIB-free medium from rinsed paired endoneurial preparations prelabeled for $10 \mathrm{~min}$ in $\left.2.5 \mathrm{mM}{ }^{3} \mathrm{H}\right] \mathrm{AIB}$ in the presence or absence of sodium for endoneurial preparations derived from either nondiabetic or untreated diabetic rabbits.

Analytical methods. Plasma glucose was determined in a Beckman Glucose Analyzer II (Beckman Instruments, Fullerton, CA). Plasma and sciatic nerve MI was determined by gas-liquid chromatography in protein-free Somogyi filtrates of venous blood plasma or sciatic nerve homogenates and expressed per milliliter of plasma or per gram wet weight of whole nerve (29). The determinations of MI were performed on trimethylsilyl derivatives of lyophilized filtrates containing alphaD-methyl mannopyranoside as an internal standard in a Varian 3700 gas-liquid chromatograph (Varian Associates, Inc., Palo Alto, CA) on a $6 \mathrm{ft} \times 4 \mathrm{~mm} \mathrm{3 \%} \mathrm{SE-30} \mathrm{Gaschrom} \mathrm{Q} \mathrm{glass} \mathrm{column} \mathrm{at} 185^{\circ} \mathrm{C}$ with a nitrogen carrier-gas flow rate of $40 \mathrm{ml} / \mathrm{min}$ using a flame ionization detector maintained at $250^{\circ} \mathrm{C}$. Standard curves were determined daily, and the recovery of added MI to nerve or plasma samples consistently exceeded $95 \%$. 
Plasma and sciatic nerve amino acids were quantitated using a Beckman Amino Acid Analyzer (model 6300; Beckman Instruments, Palo Alto, CA) equipped with a fluorometric detector. Plasma samples were precipitated using sulfosalicylic acid $(50 \mathrm{mg} / \mathrm{ml}$ plasma) and centrifuged. $500 \mu \mathrm{l}$ of each resulting supernatant were added to $1.0 \mathrm{ml}$ of $0.1 \%$ trifluoroacetic acid containing $30 \%$ methanol and filtered through a Waters Sep-Pak (Waters Division, Millipore Corp., Milford, MA) as previously described (30), and $50 \mu \mathrm{l}$ of the filtrate injected directly onto the amino acid analyzer. Nerve samples were homogenized in $10 \mathrm{vol}$ of $6 \%$ trichloracetic acid, centrifuged, and the supernatants extracted three times with equal volumes of diethylether. The final aqueous phases were lyophilized, reconstituted in $1 \mathrm{ml}$ of $0.1 \%$ trifluoroacetic acid/methanol (80:20 vol/vol), filtered through Waters Sep-Paks (30), lyophilized, reconstituted in $125 \mu \mathrm{l}$ of starting buffer for the amino acid analyzer, and small aliquots or dilutions injected. Computations were performed using the external standard method.

Statistical methods. Data were calculated as mean \pm SEM. Signifcance of difference between groups was analyzed by Student's twotailed $t$ test. In instances where comparisons were made among multiple groups, a more conservative test for significance would be to compare each $P$ value with 0.050 divided by the number of groups, which is the Bonferroni adjustment to the $P$ value. The differences between paired samples were analyzed by the $t$ test for paired comparisons. Kinetic analysis of AIB uptake was performed by the Eadie-Hofstee and Lineweaver-Burk transformations of the Michaelis-Menten equation. Linear regression analysis was performed by the method of least squares.

\section{Results}

\section{AIB uptake in endoneurial preparations from nondiabetic rabbits}

Linearity with respect to time (Fig. 1). The intracellular uptake of $2.5 \mathrm{mM}$ AIB was linear with time for $15 \mathrm{~min}$ after preincubation in AIB-free medium for $1 \mathrm{~h}$ (Fig. 1) or $2 \mathrm{~h}$ (data not shown). When medium sodium was replaced with equimolar concentrations of choline, AIB uptake remained linear with time for $15 \mathrm{~min}$, but uptake was reduced by about half. Paired 10-min incubations in medium containing sodium or choline were routinely employed to estimate the initial rate of composite (sodium-dependent + sodium-independent) and sodiumdependent AIB uptake.

Effect of medium AIB concentration (Fig. 2). Composite intracellular AIB uptake was assessed during 10-min incubations in the presence of sodium ion at AIB concentrations between 0.5 and $12.5 \mathrm{mM}$. Composite AIB uptake increased rapidly with AIB concentration below $2.5 \mathrm{mM}$, after which AIB uptake continued to increase but at a slower rate (Fig. 2, solid line). This pattern was consistent with the presence of two transport components, a higher affinity one that became fully saturated and a lower affinity one, which demonstrated little or no saturation over the AIB concentration range tested.

Effect of sodium gradient (Fig. 2 and 3, Table I). To determine which component constituted sodium-dependent cotransport, uptake of $0.5-12.5 \mathrm{mM}$ AIB was studied in the absence of extracellular sodium. The initial rate of AIB uptake increased linearly with concentration $(r=0.994, y$-intercept $=33 \mathrm{nmol} / \mathrm{kg}$ per min, slope $=253 \mathrm{nmol} / \mathrm{kg}$ per min per mM; Fig. 2, dotted line), suggesting that sodium-independent uptake did not saturate within the concentration range tested. Sodium-dependent uptake velocity (composite uptake - sodium-independent uptake) increased as a function of AIB concentration, but at a progressively decreasing rate, suggesting a saturable uptake process (Fig. 2, dashed line). Therefore,

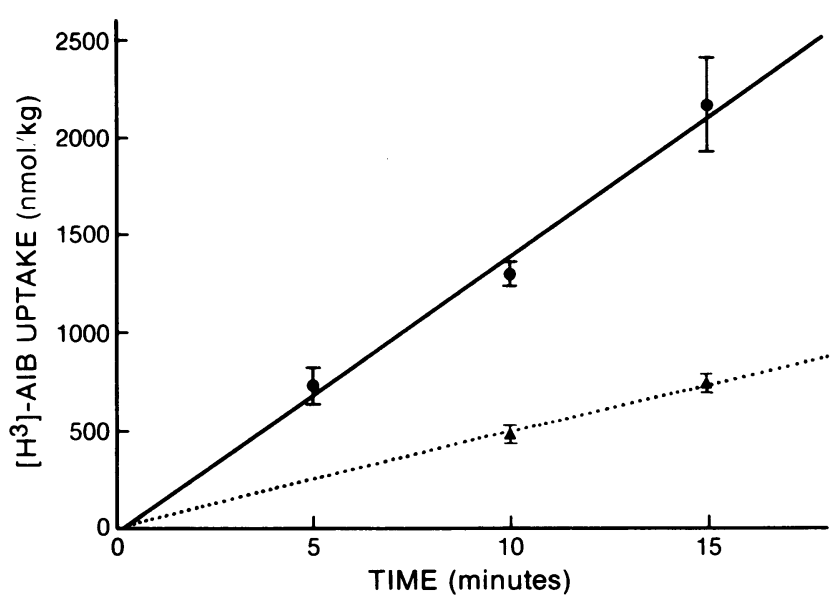

Figure 1. Endoneurial intracellular AIB uptake as a function of time. Paired endoneurial preparations were derived by microdissection and limited collagenase digestion from rabbit sciatic nerve as described in Methods, and incubated for various time periods with $2.5 \mathrm{mM}\left[{ }^{3} \mathrm{H}\right]-$ AIB and tracer concentrations of $1-\left[{ }^{14} \mathrm{C}\right]$ mannitol (as a marker for extracellular space) in KRB pH 7.4 containing $4.5 \%$ dialyzed BSA and $5 \mathrm{mM}$ glucose equilibrated with $5 \% \mathrm{CO}_{2} / 95 \% \mathrm{O}_{2}$ at $37^{\circ} \mathrm{C}$ in the presence (solid line, filled circles) or absence (dotted line, triangles) of sodium. Aliquots of neutralized perchloric acid filtrates of incubation medium and endoneurial homogenates were counted for ${ }^{3} \mathrm{H}$ and ${ }^{14} \mathrm{C}$ by liquid scintillation spectrometry, and endoneurial ${ }^{3} \mathrm{H}$ accumulation, corrected for AIB specific activity in the incubation medium and for extracellular contamination, was expressed per unit wet weight of endoneurial tissue.

AIB uptake consisted of two transport components, one sodium dependent and saturable, and the other sodium independent and unsaturable below $12.5 \mathrm{mM}$. To confirm dependency of AIB uptake on the transmembrane sodium gradient, so-

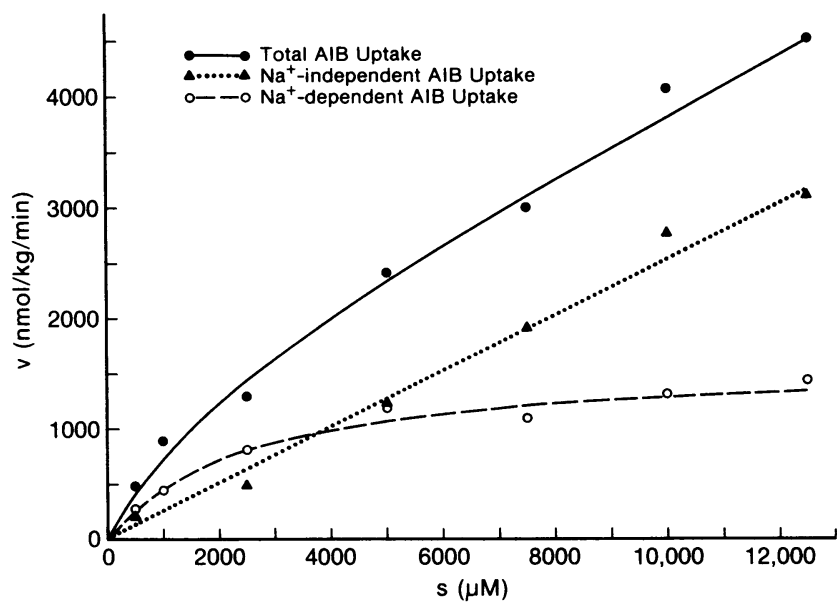

Figure 2. Initial rate of intracellular AIB uptake by endoneurial preparations derived from nondiabetic animals as a function of medium AIB concentration. Intracellular AIB uptake was measured at varying medium AIB concentrations after 10-min incubations as described in Methods in the presence (solid line, filled circles) or absence (dotted line, triangles) of sodium. Sodium-dependent uptake (dashed lines, open circles) was computed by subtracting the uptake in sodium-free medium from the composite uptake measured in the presence of sodium. $v$, rate of intracellular AIB uptake. $s$, medium AIB concentration. Each data point represents the mean of at least three determinations. 

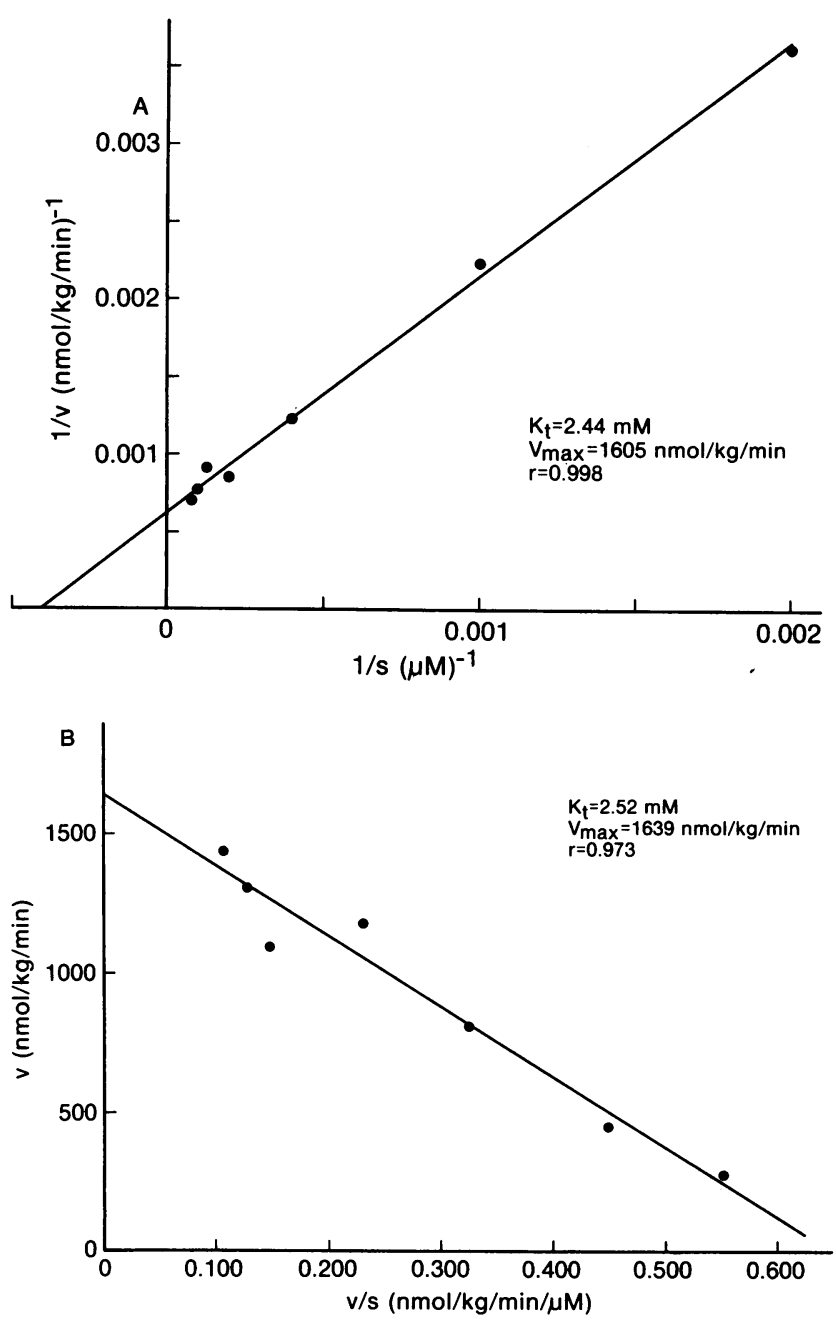

Figure 3. Sodium-dependent intracellular AIB uptake and medium AIB concentration plotted as linear transformations of the MichaelisMenten equation. $(A)$ Uptake velocity and medium AIB concentration graphed as their double reciprocals (Lineweaver-Burk transformation). (B) Uptake velocity as a function of the ratio of uptake velocity to medium AIB concentration (Eadie-Hofstee transformation). Each data point represents the mean of three or more determinations. $v$, rate of sodium-dependent AIB uptake; $s$, medium AIB concentration.

dium-dependent uptake of $2.5 \mathrm{mM}$ AIB was measured after a 10 -min preincubation with and in the presence of $2 \mathrm{mM}$ ouabain. Sodium-dependent $2.5 \mathrm{mM}$ AIB uptake was virtually completely inhibited by ouabain suggesting that AIB uptake is sodium gradient dependent (Table I), while $2.5 \mathrm{mM}$ sodiumindependent uptake was unaffected by ouabain: $691 \pm 164$ $\mathrm{nmol} / \mathrm{kg}$ per min, $n=6$, with and $724 \pm 120 \mathrm{nmol} / \mathrm{kg}$ per min, $n=7$, without $2 \mathrm{mM}$ ouabain, $P=\mathrm{NS}$.)

Although AIB is transported by the sodium-cotransport system specific for amino acids with short, polar or linear side chains ("system A"), AIB is also transported by other carriers as well in some cells (31). Therefore, the ability of excess MeAIB, a system A-specific substrate, to inhibit sodium-gradient dependent AIB uptake was explored. The uptake of 1 $\mathrm{mM}\left[{ }^{3} \mathrm{H}\right] \mathrm{AIB}$ in the presence of $10 \mathrm{mM}$ MeAIB was marginally (but statistically significantly) affected by the presence of sodium ion in the incubation medium $(239 \pm 13 \mathrm{nmol} / \mathrm{kg}$ per
Table I. Nondiabetic Endoneurial Sodium-dependent AIB Uptake (Medium AIB Concentration $2.5 \mathrm{mM}$ )

\begin{tabular}{lcc}
\hline Incubation conditions & $\begin{array}{c}\text { Sodium-dependent } \\
\text { AIB uptake }\end{array}$ & $\begin{array}{c}P \text { vs. } 5 \mathrm{mM} \\
\text { Glucose alone }\end{array}$ \\
\hline & $($ nmol/kg/min $)$ & - \\
Glucose, $5 \mathrm{mM} \mathrm{(7)}$ & $663 \pm 59$ & \\
Glucose, $20 \mathrm{mM}(5)$ & $824 \pm 159$ & $\mathrm{NS}$ \\
Ouabain, $2 \mathrm{mM}(6)$ & $101 \pm 74$ & $<0.001$ \\
Insulin, $100 \mu \mathrm{U} / \mathrm{ml}$ & $607 \pm 133$ & $\mathrm{NS}$ \\
Insulin, $1,000 \mu \mathrm{U} / \mathrm{ml}$ & $620 \pm 94$ & $\mathrm{NS}$ \\
\hline
\end{tabular}

min without sodium vs. $323 \pm 32 \mathrm{nmol} / \mathrm{kg}$ per min with sodium, mean delta of paired samples $+84 \pm 29 \mathrm{nmol} / \mathrm{kg}$ per min, $n=6, P<0.05$ ); this implies that at this AIB concentration, $30 \%$ of non-system A AIB uptake was sodium dependent. Sodium-dependent uptake of $1 \mathrm{mM}$ AIB in the absence of MeAIB was $636 \pm 141 \mathrm{nmol} / \mathrm{kg}$ per $\min (n=4)$ (Fig. 2$)$ so that residual (non-system A) sodium-dependent AIB uptake comprised only $13 \%$ of total sodium-dependent AIB uptake. Thus at least $87 \%$ of sodium-dependent $1 \mathrm{mM}$ AIB uptake would appear to occur via system A (31).

When sodium-dependent AIB uptake and medium AIB concentration were plotted as their double reciprocals (Fig. 3 $A$ ) or when uptake was plotted as a function of the ratio of uptake to medium AIB concentration (Eadie-Hofstee transformation, Fig. $3 \mathrm{~B}$ ), linear relationships were generated $(r$ $=0.998$ and 0.973 , respectively). By the Lineweaver-Burk transformation of the Michaelis-Menten equation, the apparent transport constant $\left(K_{\mathrm{t}}=\right.$ concentration for half-maximal rate of uptake) was $2.44 \mathrm{mM}$ and the apparent maximal transport velocity $\left(V_{\max }\right)$ was $1,605 \mathrm{nmol} / \mathrm{kg}$ per min. The EadieHofstee transformation of the Michaelis-Menten equation yielded values of $2.52 \mathrm{mM}$ and $1,639 \mathrm{nmol} / \mathrm{kg}$ per min, respectively, for $K_{\mathrm{t}}$ and $V_{\max }$. These kinetic data suggested that sodium-dependent AIB uptake conformed to Michaelis-Menten kinetics, hereby implying that this uptake process represented carrier-mediated sodium-dependent transport.

Effect of 14 day alloxan diabetes on endoneurial AIB uptake (Table II, Figs. 4 and 5). In order to assess the effect of experimental diabetes on nerve sodium-dependent amino acid uptake, composite and sodium-independent uptake was measured in endoneurial preparations derived from rabbits with untreated alloxan diabetes of $14 \mathrm{~d}$ duration. Untreated alloxan diabetes was associated with significant reductions in body weight and sciatic nerve MI content, elevation in fasting plasma glucose concentration, but no change in fasting plasma MI content (Table II, lines 1 and 2). Composite AIB uptake was generally reduced over the concentration tested, while sodium-independent AIB uptake was essentially intact (Fig. 4). The selective effect of experimental diabetes on the sodiumdependent component of AIB uptake was confirmed in paired experiments with a medium AIB concentration of $2.5 \mathrm{mM}$ (Fig. 5). Sodium-dependent AIB uptake in diabetic endoneurial preparations approximated classical Michaelis-Menten kinetics only in the middle concentration ranges tested (2.5-7.5 $\mathrm{mM}$ ), where the Lineweaver-Burk and Eadie-Hofstee transformations yielded values of $1.89 \mathrm{mM}$ and $509 \mathrm{nmol} / \mathrm{kg}$ per 
Table II. Effect of Alloxan Diabetes, MI Supplementation, and Sorbinil Administration on Body Weight, Plasma Glucose, and Plasma and Sciatic Nerve MI

\begin{tabular}{|c|c|c|c|c|}
\hline Animal/treatment & Body weight & Plasma glucose & Plasma MI & Nerve MI \\
\hline & $g$ & $m g / d L$ & $\mu M$ & $\mathrm{mmol} / \mathrm{kg}$ \\
\hline \multirow[t]{2}{*}{ Nondiabetic } & $253 \pm 77$ & $116 \pm 57$ & $47.9 \pm 2.7$ & $\left.\Gamma^{5.49 \pm 0.23}\right\rceil$ \\
\hline & $P<0.001$ & $P<0.001$ & & $P<0.025$ \\
\hline \multirow[t]{2}{*}{ Diabetic } & $200 \pm 6-$ & $467 \pm 13-$ & $46.9 \pm 3.0$ & $4.57 \pm 0.25$ \\
\hline & & & & $P<0.010$ \\
\hline Diabetic + MI & $183 \pm 12$ & لـ & $56.8 \pm 10.6$ & $L_{4.10 \pm 0.33}$ \\
\hline
\end{tabular}

min, and $1.91 \mathrm{mM}$ and $517 \mathrm{nmol} / \mathrm{kg}$ per min, respectively for apparent $K_{\mathrm{t}}$ and $V_{\max }$, though the reliability of these values extrapolated from such a small concentration range are questionable.

Effects of elevated ambient glucose concentration and insulin on endoneurial sodium-dependent AIB uptake (Table I). Because experimental diabetes perturbs both plasma insulin and glucose concentrations, the acute effects of both elevated ambient glucose concentration, and physiologic or supraphysiologic concentrations of insulin on endoneurial AIB uptake were tested. Nondiabetic endoneurial preparations were preincubated and incubated in the presence of $20 \mathrm{mM}$ glucose, or 100 or $1,000 \mu \mathrm{U} / \mathrm{ml}$ insulin (crystalline porcine; Eli Lilly

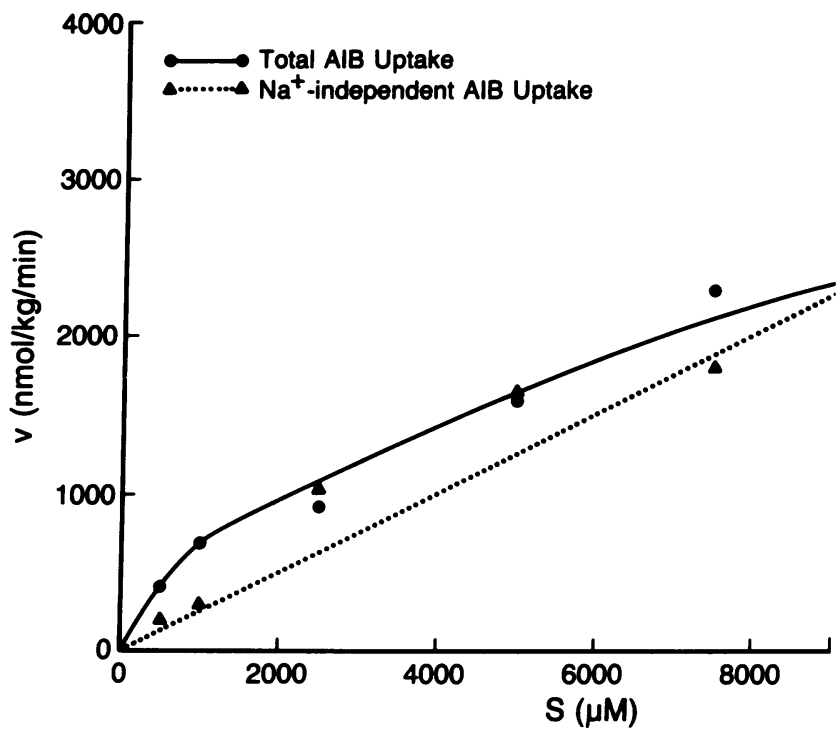

Figure 4. Initial rate of intracellular AIB uptake by endoneurial preparations derived from 14-d alloxan-diabetic animals as a function of medium AIB concentration. Composite (solid line, filled circles) and sodium-dependent (triangles) AIB uptake was measured, and sodium-independent AIB uptake (not shown) computed as described in the previous figure. Apparent sodium-dependent uptake, which became negative at high AIB concentrations, was not graphed for clarity. The regression line for sodium-independent uptake in non-diabetic endoneurial preparations from Fig. 2 is reproduced for comparison. $v$, rate of intracellular AIB uptake. $s$, medium AIB concentration. Each data point represents the mean of at least three determinations.
Co., Indianapolis, IN) and AIB uptake measured. Neither elevated ambient glucose concentration nor insulin acutely effected sodium-dependent AIB uptake (Table I).

Effect of MI supplementation and sorbinil administration on endoneurial AIB uptake in alloxan diabetes (Tables II, Figs. 5 and 6). Acute spontaneous or streptozotocin-induced diabetes in rats, and alloxan-induced diabetes in rabbits, produce a $25-50 \%$ reduction in sodium-plus-potassium-stimulated and/or ouabain-sensitive ATPase activities in homogenates or axolemmal-enriched membrane fractions derived from sciatic nerve (2). These reductions in nerve sodium-potassium ATPase activity associated with acute spontaneous or experimental diabetes are prevented by either MI supplementation or aldose reductase inhibitor administration in vivo (2) or reversed by protein kinase-C agonists in vitro (4-6). Therefore the effects of MI supplementation or sorbinil administration on diabetic nerve AIB uptake were tested. Sorbinil raised

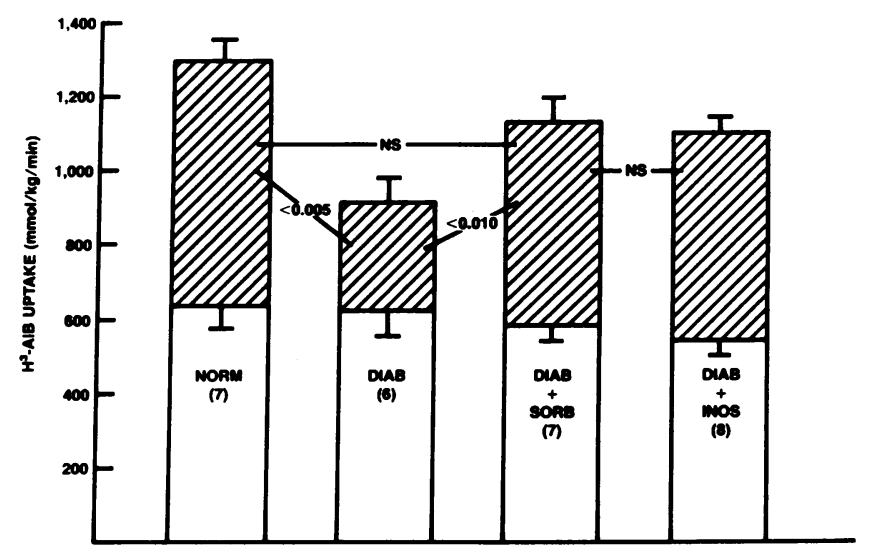

Figure 5. Composite and sodium-dependent (hatched bar) AIB uptake in endoneurial preparations from nondiabetic controls, untreated alloxan diabetic animals, and MI-treated and sorbinil-treated alloxan diabetic animals. Medium AIB concentration $2.5 \mathrm{mM}$. Rabbits were made alloxan diabetic as described in Methods and Table II. Endoneurial preparations were prepared, preincubated and exposed to AIB containing medium as described in Methods. Composite uptake refers to the initial rate of intracellular AIB uptake in the presence of sodium ion, and sodium-dependent uptake refers to the difference between the initial rate of AIB uptake in the presence and absence of sodium. Error bars indicate standard error of the mean for composite uptake (upper error bar) and sodium-dependent uptake (lower error bar). 


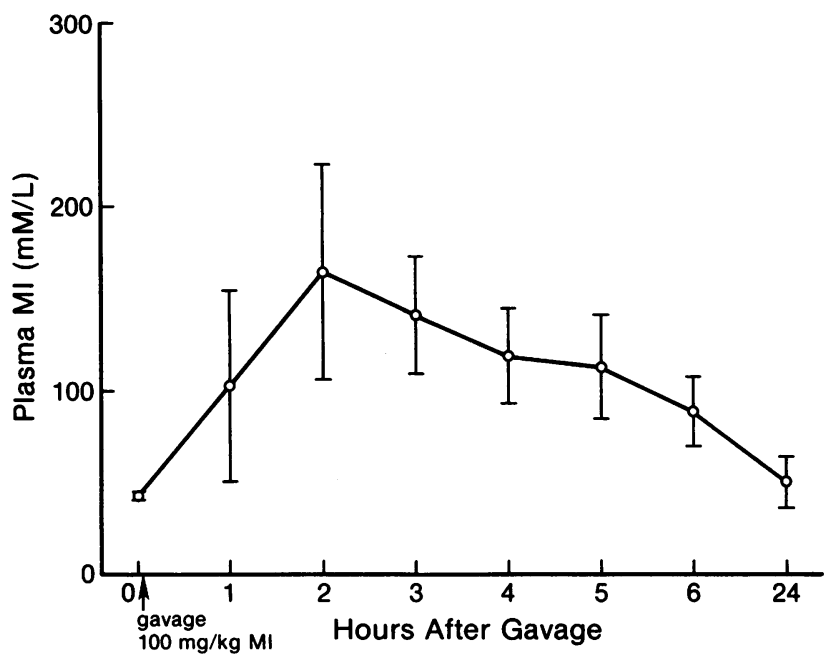

Figure 6. Plasma MI concentration as a function of time after administration of a single $100 \mathrm{mg} / \mathrm{kg}$ dose of MI by gavage to normal fasted rabbits.

nerve MI but did not affect body weight, plasma glucose, or plasma MI in alloxan diabetic rabbits (Table II, line 3). Sodium-dependent AIB uptake in endoneurial preparations from alloxan diabetic animals treated with sorbinil was no longer significantly different from that of nondiabetic controls (Fig. 5). MI supplementation did not alter body weight or plasma glucose in alloxan diabetic rabbits and raised neither nerve MI, nor plasma MI after an overnight fast (Table II, line 4). However, MI supplementation similarly and significantly raised composite and sodium-dependent AIB uptake in the presence of $2.5 \mathrm{mM}$ AIB (Fig. 5). To better define the action on AIB uptake of MI supplementation that altered neither fasting plasma nor nerve MI, plasma MI was monitored frequently after administration of $100 \mathrm{mg} / \mathrm{kg}$ MI by gavage to nondiabetic rabbits (Fig. 6). Peak plasma MI concentration $2 \mathrm{~h}$ after gavage was not statistically significantly lower than that which normalized sciatic nerve MI level and sodium-potassium ATPase in streptozotocin-diabetic rats fed 1\% MI diets (2), but plasma MI returned to baseline in the rabbit within $24 \mathrm{~h}$ after gavage with MI (Fig. 6). Thus gavage with $100 \mathrm{mg} / \mathrm{kg} \mathrm{MI}$ in the rabbit reproduced the plasma concentrations resulting from $1 \% \mathrm{MI}$ diets in the rat, but only transiently. Daily gavage with a 10-fold higher dose of MI did correct sciatic nerve MI content $(5.73 \pm 0.64 \mathrm{mmol} / \mathrm{kg}, n=6, P=\mathrm{ns}$ vs. non-diabetic [Table II, line 1 , column 4].)

Effect of alloxan diabetes on plasma and sciatic nerve amino acid levels (Table III). Experimental diabetes raised the plasma concentration of the branched-chain amino acids (BCAA's: leucine, isoleucine and valine) and aspartate, and lowered the concentration of alanine, while producing opposite trends in the concentrations of these amino acids in sciatic nerve (Table III). Therefore, the inhibition of sodium-dependent AIB uptake by diabetes could not be readily attributed to a "trans effect" resulting from increased intracellular levels of amino acids with short, polar or linear side chains (31).

Effect of in vitro exposure to PMA on diabetic endoneurial AIB uptake. To determine if normalization of sodium-potassium ATPase activity in diabetic nerve rapidly corrected sodium-gradient dependent AIB uptake, endoneurial AIB up-
Table III. Effect of Alloxan Diabetes on Plasma and Sciatic Nerve Amino Acid Levels

\begin{tabular}{lccccc}
\hline & \multicolumn{2}{c}{ Plasma $(\mu M)$} & & \multicolumn{2}{c}{ Nerve $(\mu m o l / k g)$} \\
\cline { 2 - 3 } \cline { 5 - 6 } & Control & Diabetic & & Control & Diabetic \\
\hline Tryptophan & $35 \pm 6$ & $43 \pm 5$ & & $207 \pm 25$ & $191 \pm 17$ \\
Tyrosine & $66 \pm 5$ & $52 \pm 6$ & & $19 \pm 12$ & $10 \pm 2$ \\
Phenylalanine & $46 \pm 3$ & $49 \pm 6^{*}$ & & $20 \pm 6$ & $12 \pm 2$ \\
Leucine & $108 \pm 10$ & $349 \pm 63^{*}$ & & $42 \pm 12$ & $25 \pm 3$ \\
Isoleucine & $54 \pm 3$ & $162 \pm 25^{\ddagger}$ & & $20 \pm 7$ & $9 \pm 2$ \\
Valine & $191 \pm 1$ & $547 \pm 78$ & & $35 \pm 13$ & $15 \pm 1$ \\
Aspartate & $23 \pm 1$ & $15 \pm 3^{8}$ & & $603 \pm 51$ & $578 \pm 41$ \\
Glutamate & ND & ND & & $967 \pm 43$ & $1,086 \pm 148$ \\
Threonine & $82 \pm 18$ & $122 \pm 43$ & & $85 \pm 11$ & $120 \pm 19$ \\
Serine & $157 \pm 58$ & $79 \pm 11$ & & $299 \pm 76$ & $244 \pm 41$ \\
Glycine & $289 \pm 37$ & $414 \pm 142$ & & $474 \pm 92$ & $361 \pm 55$ \\
Alanine & $398 \pm 48$ & $205 \pm 41^{11}$ & & $139 \pm 24$ & $245 \pm 47$ \\
Lysine & $158 \pm 17$ & $136 \pm 24$ & & ND & ND \\
Histidine & $98 \pm 10$ & $60 \pm 17$ & & ND & ND \\
Arginine & $347 \pm 133$ & $179 \pm 2$ & & ND & ND \\
& & & & \\
\hline
\end{tabular}

Since 15 different amino acids were measured in each group, a more conservative test for significance would be to compare each $P$ value with 0.050 divided by 15 or 0.003 , which is the Bonferroni adjustment to the $P$ value.

ND, not detectable. ${ }^{*} P<0.005,{ }^{\ddagger} P<0.001,{ }^{8} P<0.050, " 1 P<0.025$

take was compared after a 1-h incubation in the presence and absence of PMA in paired experiments with control incubations receiving $10 \mu \mathrm{l}$ of the DMSO vehicle alone. Neither PMA nor DMSO altered sodium-dependent AIB uptake in diabetic endoneurial preparations (DMSO control $=346 \pm 214,+$ PMA $=432 \pm 185$, mean difference of pairs $+87 \pm 220 \mathrm{nmol} / \mathrm{kg}$ per $\min , n=5, P=\mathrm{NS}$ ).

\section{Discussion}

Metabolically stable endoneurial preparations comprised largely of axons and Schwann cells (28) from rabbit sciatic nerve exhibit sodium-gradient dependent saturable uptake of the nonmetabolized amino acid analogue AIB. This presumed carrier-mediated sodium cotransport system for amino acids with short, polar or linear side chains (system A) (31) conforms to Michaelis-Menten kinetics. The apparent $K_{\mathrm{t}}$ of $2.5 \mathrm{mM}$ approximates that of similar amino acid transport systems in other tissues (32) including peripheral nerve (33). 2 wk of untreated experimental diabetes that does not interfere with in vitro endoneurial metabolic stability (27) reduces nerve $\mathrm{MI}$ content and sodium-potassium ATPase activity (6), and markedly depresses endoneurial sodium-gradient dependent (but not sodium-independent) AIB uptake over a concentration range extending fivefold above and below the apparent $K_{t}$. Decreased AIB uptake cannot be explained on the basis of a "trans effect" due to increased intraneural concentrations of system A-transported amino acids, or by alterations in AIB efflux since sodium-dependent AIB uptake was essentially unidirectional during the brief experimental periods employed in these studies. Neither hyperglycemic concentrations of glucose nor physiologic or supraphysiologic concentrations of insulin acutely affect AIB uptake in vitro in nondiabetic endoneurial 
preparations at the $K_{\mathrm{t}}$. Administration of an aldose reductase inhibitor that normalizes nerve MI levels corrects the reduction in sodium-gradient dependent AIB uptake after 2 wk of experimental diabetes. MI supplementation (that in the diabetic rat does not alter nerve polyol pathway intermediates [29]) also corrects sodium-gradient dependent AIB uptake, implicating MI depletion in this transport defect in diabetic peripheral nerve. However, the MI supplementation that reproduced the effect of sorbinil on sodium-dependent AIB uptake did not significantly raise the overall tissue MI content of diabetic rabbit sciatic nerve. As discussed below, this apparent paradox is consistent with the concept of Winegrad and coworkers who postulate that depletion or repletion of a specific small metabolically labile pool of tissue MI which is poorly reflected by changes in overall tissue MI content may be particularly relevant to abnormalities in membrane transport in diabetic tissues $(8,26)$. This concept was recently extended to renal tubular transport by the suggestion that impaired renal tubular sodium-dependent ascorbate transport in the streptozotocin-diabetic rat is corrected by dietary MI supplementation without any concomitant change in renal cortical or medullary MI content (34).

Diabetes raises plasma BCAA levels in the rat (35), man (36), and the rabbit. The parallel increases in plasma and brain BCAA in the diabetic rat (35) contrast with the paradoxical decrease in diabetic rabbit peripheral nerve BCAA level, though this difference did not attain statistical significance. While species effects cannot be excluded, these divergent responses in brain and peripheral nerve BCAA's to the raised plasma BCAA levels in diabetes more likely reflects a fundamental differences in brain and nerve amino acid transport: system A transport occurs in peripheral nerve but not at the blood-brain barrier (37) where sodium-independent bidirectional system $L$ would facilitate equilibration of brain and plasma BCAA levels, while the uptake of other large neutral amino acids would be reduced in diabetes due to competitive inhibition by BCAA's (37). In peripheral nerve, active systemA uptake would greatly exceed influx via the bidirectional system L, which would promote efflux of amino acids transported in common and competitively by both systems (38). (The failure to detect a fall in system A amino acid content despite diminished AIB uptake in diabetic nerve may reflect the large tissue pool of system A amino acids [38].) System L amino acids (BCAA's, tyrosine and phenylalanine) tended to be lower in diabetic nerve, though not statistically significantly so, consistent with a reduction in system $\mathrm{A}$ amino acid transport and the much smaller tissue pool size of system $L$ amino acids. Thus the observed effects of diabetes on amino acid content in diabetic rabbit nerve are not inconsistent with a significant reduction in vivo in sodium-dependent system $\mathrm{A}$ amino acid transport in diabetic nerve.

MI regulates intracellular metabolism primarily through its incorporation into inositol-containing membrane phospholipids that serve as precursors for phospholipase-C-generated inositol-phosphate and diacylglycerol second messengers (39). The known relationship(s) between phosphoinositide signaling and membrane transport are widespread and complex, involving highly regulated inositol-1,4,5-trisphosphate- and inositol-1,4,5-tetrakisphosphate-mediated intracellular $(40,41)$ and transplasmalemmal (40) calcium fluxes, and diacyl-glycerol-mediated stimulation of phospholipid-and-calcium-dependent protein kinase $C$ (39). Protein kinase $C$ agonists affect growth and differentiation in some cells, and have multiple effects on membrane transport such as the induction of the glucose transporter gene (42). In LLC-PK ${ }^{1}$ cells, PKC-mediated activation of system $A$ sodium-dependent amino acid transport is demonstrable primarily in confluent cells with constitutively low amino acid uptake but not in dividing cells with constitutively high amino acid transport (43). The sorbinil- and MI-sensitive defect in amino acid transport in diabetic peripheral nerve may be a direct consequence of the estimated fourfold rise in intraaxonal sodium (if the AIB transport defect resides in the axonal compartment), or a similar reduction in the transmembrane electrochemical sodium-gradient in the Schwann cell, both of which may reflect impaired sodium-potassium ATPase activity (3). Alternatively, MI depletion in diabetic nerve may independently reduce sodiumpotassium ATPase activity and sodium-dependent AIB uptake, the latter possibly via an indirect mechanism analogous to that described in quiescent LLC-PK ${ }^{1}$ cells (43). The fact that PMA, a protein kinase $\mathrm{C}$ agonist that rapidly corrects the MIrelated reduction in sodium-potassium ATPase in diabetic rabbit and rat nerve (4-6), does not acutely improve sodiumgradient dependent AIB uptake in vitro in endoneurial preparations from diabetic rabbits implies that these two processes are independent, or that the reestablishment of the normal electrochemical sodium gradient is delayed once sodium-potassium ATPase activity is restored. A third possibility, that impaired sodium-gradient dependent AIB uptake in diabetic peripheral nerve represents a consequence of MI depletion at some distant site (perhaps the anterior horn or dorsal root ganglia neuronal cell body) that is fully corrected by MI-supplementation, cannot be rigorously excluded. In any case, both sodium-dependent AIB uptake and sodium-potassium ATPase activity appear to be reduced in diabetic nerve as a result of underlying MI depletion. These two defects may be linked serially or in parallel, and may involve one or more metabolic pools of MI, and/or distinct but phosphoinositide-related processes (39). All of these possibilities imply that metabolism of a specific MI pool or pools rather than the total tissue MI content mediate some of the effects of MI depletion and repletion on nerve metabolism such as the defect in system A amino transport, as has been suggested by Winegrad and his colleagues $(8,26)$.

Although rapidly reversible slowing of nerve conduction in human $(9,44)$ and animal $(45)$ diabetes theoretically may be attributable in part to acute alterations in nerve MI metabolism, most but not all slowing of nerve conduction in established diabetes and diabetic neuropathy is thought to reflect nerve fiber loss, atrophy, and/or demyelination (46). Reduced leucine incorporation into myelin in diabetic rodent nerve has been postulated to be a contributing factor to peripheral nerve hypomyelination in diabetes $(47,48)$. This defect may reflect impaired amino acid uptake by diabetic peripheral nerve in vitro (while this defect appeared to be partially insulin sensitive, pharmocological concentrations of insulin far in excess of those employed in the present report were required (46)). Reduced amino acid uptake by dorsal root ganglia from the streptozotocin diabetic rat is associated with diminished sodium-potassium ATPase activity, and has been invoked in the accompanying axonal "dwindling" or "atrophy" $(49,50)$. Hence chronically reduced MI- and sorbinil-responsive sodium-dependent amino acid uptake in axons and/or Schwann cells may be important links between acute metabolic derange- 
ments and chronic structural defects in diabetic nerve, thereby providing a plausible mechanism by which longstanding alterations in nerve MI metabolism induced by hyperglycemia might contribute to the pathogenesis of the structural hallmarks of chronic diabetic neuropathy.

In summary, important polyol-pathway related abnormalities in MI metabolism in diabetic peripheral nerve, such as that responsible for impaired sodium-amino acid cotransport, may be corrected by MI supplementation that fails to detectably alter the concurrent decrease in whole-nerve MI content. This most likely reflects the fact that the metabolically active, physiologically important MI pools in the peripheral nervous system may represent only a small fraction of, and/or be poorly reflected by, the composite tissue MI content of peripheral nerve. Therefore, the presence or absence of alterations in whole tissue MI content in response to diabetes or metabolic intervention are poor indicators of the presence or absence of physiologically and perhaps pathogenetically important abnormalities in MI or phosphoinositide metabolism.

\section{Acknowledgments}

The authors gratefully acknowledge the technical assistance of Carol Korbanic, Barbara Thornton, and Lorraine Weber, and advice of the Biostatistics Core of the Michigan Diabetes Research and Training Center. This research was supported in part by the U. S. Public Health Service (RO1-DK26862 and DK38304 [Dr. Greene], and HD-24730 [Dr. Fernstrom]), the American Diabetes Association (Dr. Finegold) and the Harry Soffer Memorial Research Fund of the University of Pittsburgh (Dr. Greene).

\section{References}

1. Committee on Health Care Issues, American Neurological Association. 1986. Does improved control of glycemia prevent or ameliorate diabetic neuropathy? Ann. Neurol. 19:288-290.

2. Greene, D. A., S. A. Lattimer, and A. A. F. Sima. 1988. Pathogenesis and prevention of diabetic neuropathy. Diabetes/Metabolism Rev. 4:201-221.

3. Brismar, T., A. A. F. Sima, and D. A. Greene. 1987. Reversible and irreversible nodal dysfunction in diabetic neuropathy. Ann. Neurol. 21:504-507.

4. Kim, J., and D. A. Greene. 1987. Correction of the myo-inositol-related $\mathrm{Na} / \mathrm{K}-\mathrm{ATPase}$ defect in axolemmal-enriched, protein kinase C-containing isolated membranes from diabetic rat peripheral nerve by phorbol myristate. Clin. Res. 35:624A.

5. Lattimer, S. A., and D. A. Greene. 1987. Protein kinase C agonists rapidly correct impaired $\mathrm{Na} / \mathrm{K}$-ATPase in diabetic nerve in vitro. Diabetes. 36:75A. (Abstr.)

6. Lattimer, S. A., A. A. F. Sima, and D. A. Greene. 1989. Rapid in vitro correction of impaired $(\mathrm{Na}, \mathrm{K})$-ATPase in diabetic peripheral nerve by protein kinase-C agonists: suggested phosphoinositide regulation and integration of neural (Na,K)-ATPase. Am. J. Physiol. 256 (Endocrinol. Metab. 19): E264-E269.

7. Greene, D. A., S. A. Lattimer, and A. A. F. Sima. 1987. Sorbitol, phosphoinositides and the sodium-potassium ATPase in the pathogenesis of diabetic complications. N. Engl. J. Med. 316:599-606.

8. Winegrad, A. I. 1987. Banting lecture 1986. Does a common mechanism induce the diverse complications of diabetes? Diabetes. 36:396-406.

9. Judzewitsch, R. G., J. B. Jaspan, K. S. Polonsky, C. R. Weinberg, J. B. Halter, E. Halar, M. A. Pfeifer, C. Vukadinovic, L. Bernstein, M. Schneider, et al. 1983. Aldose reductase inhibition improves nerve conduction velocity in diabetic patients. N. Engl. J. Med. 308:119125.
10. Sima, A. A. F., V. Bril, V. Nathaniel, T. A. J. McEwen, M. B. Brown, S. A. Lattimer, and D. A. Greene. 1988. Regeneration and repair of myelinated fibers in sural nerve biopsies from patients with diabetic neuropathy treated with sorbinil, an investigational aldose reductase inhibitor. $N$. Engl. J. Med. 319(9):548-555.

11. Dyck, P. J., B. R. Zimmerman, T. H. Vilen, S. R. Minnerath, J. L. Karnes, J. K. Yao, and J. F. Poduslo. 1988. Nerve glucose, fructose, sorbitol, myo-inositol, and fiber degeneration and regeneration in diabetic neuropathy. 319:542-548.

12. Salway, J. G., J. A. Finnegan, D. Barnett, L. Whitehead, A. Karunanayka, and R. B. Payne. 1978. Effect of myoinositol on peripheral nerve function in diabetes. Lancet. ii:1282-1294.

13. Clements, R. S., Jr., B. Vourganti, T. Kuba, S. J. Oh, and B. Darnell. 1979. Dietary myo-inositol intake and peripheral nerve function in diabetic neuropathy. Metab. Clin. Exp. 28:477-483.

14. Greene, D. A., M. J. Brown, S. N. Braunstein, S. S. Schwartz, A. K. Asbury, and A. I. Winegrad. 1981. Comparison of clinical course and sequential electrophysiological tests in diabetics with symptomatic polyneuropathy and its implications for clinical trials. Diabetes. 30:139-147.

15. Clements, R. S., Jr. 1985. Review of myoinositol and sorbinil studies. Clin. Physiol. 5(Suppl. 5):90-93.

16. Gregerson, G., H. Borsting, P. Theil, and C. Servo. 1978. Myoinositol and function of peripheral nerve in human diabetics: a controlled clinical trial. Acta Neurol. Scand. 58:241-248.

17. Gregersen, G., B. Bertelsen, H. Harbo, E. Larsen, J. R. Anderson, A. Helles, M. Schmiegelow, and J. E. J. Christensen. 1983. Oral supplementation of myo-inositol: effects on peripheral nerve function in human diabetics and on the concentration in plasma, erythrocytes, urine and muscle tissue in human diabetics and normals. Acta Neurol. Scand. 67:164-172.

18. Ward, J. D. 1973. The polyol pathway in the neuropathy of early diabetes. In Early Diabetes. R. A. Camerini-Davalos and H. S. Cole, editors. Academic Press, New York. 425-432.

19. Mayhew, J. A., K. R. W. Gillon, and J. N. Hawthorne. 1983. Free and lipid inositol, sorbitol and sugars obtained post mortem from diabetic and control subjects. Diabetologia. 24:13-15.

20. Dyck, P. J., W. R. Sherman, L. M. Hallcher, F. J. Service, P. C. O'Brien, L. A. Grina, P. J. Palumbo, and C. J. Swanson. 1980. Human diabetic endoneurial sorbitol, fructose and myo-inositol related to sural nerve morphometry. Ann. Neurol. 8:590-596.

21. Greene, D. A., V. Bril, S. A. Lattimer, and A. A. F. Sima. 1987. Correction of myo-inositol depletion in diabetic human sural nerve by treatment with an aldose reductase inhibitor. Diabetes. 36(Suppl. 1):86A. (Abstr.)

22. Asbury, A. K. 1988. Understanding diabetic neuropathy. Editorial. N. Engl. J. Med. 319:577-578.

23. Dyck, P. J., B. R. Zimmerman, T. H. Vilen, S. R. Minnerath, J. L. Karnes, J. K. Yao, and J. F. Poduslo. 1988. Letter to the Editor. $N$. Engl. J. Med. 320:59.

24. Winegrad, A. I., D. A. Simmons, and D. B. Martin. 1988. Letter to the Editor. N. Engl. J. Med. 320:57-58.

25. Sima, A. A. F., and D. A. Greene. 1988. Letter to the Editor. $N$. Engl. J. Med. 320:58-60.

26. Simmons, D. A., E. F. O. Kern, A. I. Winegrad, and D. B. Martin. 1986. Basal phosphatidylinositol turnover controls aortic $\mathrm{Na}^{+} / \mathrm{K}^{+}$-ATPase. J. Clin. Invest. 77:503-513.

27. Greene, D. A., and Winegrad, A. I. 1981. Effects of acute experimental diabetes on composite energy metabolism in peripheral nerve axons and Schwann cells. Diabetes. 30:967-974.

28. Greene, D. A., A. I. Winegrad, J. L. Carpentier, M. J. Brown, M. Fukuma, and L. Orci. 1979. Rabbit sciatic nerve fascicle and 'endoneurial' preparations for in vitro studies of peripheral nerve glucose metabolism. J. Neurochem. 33:1007-1018.

29. Greene, D. A., P. V. DeJesus, and A. I. Winegrad. 1975. Effect of insulin and dietary myoinositol on impaired peripheral motor nerve conduction velocity in acute streptozotocin diabetes. J. Clin. Invest. 55:1326-1336. 
30. Fernstrom, J. D., M. S. Fernstrom, P. E. Grubb, and E. A. Volk. 1985. Absence of chronic effects of dietary protein content on brain tryptophane concentrations in rats. J. Nutr. 115:1337-1444.

31. Shotwell, M. A., M. S. Kilberg, and D. L. Oxender. 1983. The regulation of neutral amino acid transport in mammalian cells. Biochem. Biophys. Acta. 737:267-284.

32. Kilberg, M., M. E. Handlogten, and H. N. Christensen. 1980. Characteristics of an amino acid transport system in rat liver for glutamine, asparagine, histidine and closely related analogs. J. Biol. Chem. 255:4011-4019.

33. Wheeler, D. D. 1975. Amino acid transport in peripheral nerve: specificity of uptake. J. Neurochem. 24:97-104.

34. Yue, D. K., S. McLennan, E. Fisher, S. Heffernan, C. Capogreco, G. R. Ross, and J. R. Turtle. 1989. Ascorbic acid metabolism and polyol pathway in diabetes. Diabetes. 38:257-261.

35. Crandall, E. A., and J. D. Fernstrom. 1983. Effect of experimental diabetes on the levels of aromatic and branched-chain amino acids in rat blood and brain. Diabetes. 32:222-230.

36. Felig, P. 1975. Amino acid metabolism in man. Annu. Rev. Biochem. 44:933-955.

37. Pardridge, W. M. 1977. Regulation of amino acid availability to the brain. In Nutrition and the Brain. Vol. 1. R. J. Wurtman and J. J. Wurtman, editor. Raven Press, New York. 141-204.

38. Christensen, H. N. 1987. Role of membrane transport in interorgan amino acid flows: where do the depleted amino acids go in phenylketonuria? In Amino Acids in Health and Disease: New Perspectives. S. Kaufman, editor. Alan R. Liss, New York. 1-17.

39. Hawthorne, J. N. 1988. Phosphoinositides and metabolic control: how many messengers? Biochem. Soc. Trans. 16:657-660.

40. Berridge, M. J. 1988. Inositol lipids and calcium signalling. Proc. R. Soc. Lond. B. 234:359-378.
41. Danoff, S. K., S. Supattapone, and S. H. Synder. 1988. Characterization of a membrane protein from brain mediating the inhibition of inositol 1,4,5-trisphosphate receptor binding by calcium. Biochem. J. 254:701-705.

42. Hiraki, Y., O. M. Rosen, and M. J. Birnbaum. 1988. Growth factors rapidly induce expression of the glucose transporter gene. $J$. Biol. Chem. 263:13655-13662.

43. Dawson, W., and J. S. Cook. 1987. Parallel changes in amino acid transport and protein kinase C localization in LLC-PK ${ }^{1}$ cells treated with TPA or diradylglycerols. J. Cell. Physiol. 132:104-110.

44. Troni, W., Q. Carta, R. Cantello, M. T. Caselle, and I. Rainero. 1984. Peripheral nerve function and metabolic control in diabetes mellitus. Ann. Neurol. 16:178-183.

45. Greene, D. A., S. Yagihashi, S. A. Lattimer, and A. A. F. Sima. 1984. Nerve Na+-K+-ATPase, conduction and myo-inositol in the insulin deficient BB rat. Am. J. Physiol. 247:E534-E539.

46. Behse, F., F. Buchthal, and F. Carlsen. 1977. Nerve biopsy and conduction studies in diabetic neuropathy. J. Neurol. Neurosurg. Psychiatry. 40:1072-1082.

47. Spritz, N., H. Singh, and B. Marman. 1975. Metabolism of peripheral nerve myelin in experimental diabetes. J. Clin. Invest. 55:1049-1056.

48. Spritz, N., H. Singh, and B. Marman. 1975. Decrease in myelin content of rabbit sciatic nerve with aging and diabetes. Diabetes. 24:680-683.

49. Thomas, P. K., D. W. Wright, and E. Tzebelikos. 1984. Amino acid uptake by dorsal root ganglia from streptozotocin-diabetic rats. $J$. Neurol. Neurosurg. Psychiatr. 47:912-916.

50. Green, R. J., R. H. M. King, P. K. Thomas, and D. N. Baron. 1985. Sodium-potassium ATPase activity in the dorsal root ganglia of rats with streptozotocin-induced diabetes. Diabetologia. 28:104-107. 